This manuscript has been accepted for publication in Science. This version has not undergone final editing. Please refer to the complete version of record at http://www.sciencemag.org/. The manuscript may not be reproduced or used in any manner that does not fall within the fair use provisions of the Copyright Act without the prior, written permission of AAAS.

This is the author's version of the work. It is posted here by permission of the AAAS for personal use, not for redistribution. The definitive version was published in Science Magazine 23 January 2015 [DOI:10.1126/science.aaa0571] 


\section{Birth of a comet magnetosphere: a spring of water ions}

Authors: Hans Nilsson ${ }^{1,2}$, Gabriella Stenberg-Wieser ${ }^{1}$, Etienne Behar ${ }^{1,2}$, Cyril Simon Wedlund ${ }^{3}$, Herbert Gunell ${ }^{4}$, Masatoshi Yamauchi ${ }^{1}$, Rickard Lundin ${ }^{1}$, Stas Barabash $^{1}$, Martin Wieser ${ }^{1}$, Chris Carr $^{5}$, Emanuele Cupido ${ }^{5}$, Jim Burch ${ }^{6}$, Andrei Fedorov ${ }^{7}$, Jean-André Sauvaud ${ }^{7}$, Hannu Koskinen $^{8,9}$, Esa Kallio ${ }^{3,9}$, Jean-Pierre Lebreton ${ }^{10}$, Anders Eriksson ${ }^{11}$, Niklas Edberg ${ }^{11}$, Ray Goldstein ${ }^{4}$, Pierre Henri ${ }^{10}$, Christoph Koenders ${ }^{12}$, Prachet Mokashi ${ }^{6}$, Zoltan Nemeth ${ }^{13}$, Ingo Richter $^{12}$, Karoly Szego $^{13}$, Martin Volwerk ${ }^{14}$, Claire Vallat ${ }^{15}$, Martin Rubin ${ }^{16}$

\section{Affiliations:}

${ }^{1}$ Swedish Institute of Space Physics, Box 812, 98128 Kiruna, Sweden.

${ }^{2}$ Also at: Luleå University of Technology, Department of Computer Science, Electrical and Space Engineering, Rymdcampus 1, 98128 Kiruna, Sweden

${ }^{3}$ Aalto University, School of Electrical Engineering, Department of Radio Science and Engineering, P.O. Box 13000, FI-00076 Aalto, Finland.

${ }^{4}$ Belgian Institute for Space Aeronomy, Avenue Circulaire 3, 1180 Brussels, Belgium.

${ }^{5}$ Imperial College London, Exhibition Road, London SW7 2AZ, UK.

${ }^{6}$ Southwest Research Institute, 6220 Culebra Rd., San Antonio, TX 78238, USA.

${ }^{7}$ Institut de Recherche en Astrophysique et Planétologie, Toulouse, France.

${ }^{8}$ University of Helsinki, Department of Physics, P.O.Box 64, FI-00014 University of Helsinki, Finland.

${ }^{9}$ Finnish Meteorological Institute, P.O. BOX 503, FI-00101 Helsinki,Finland.

${ }^{10}$ Laboratoire de Physique et Chimie de l'Environnement et de l'Espace (LPC2E), UMR 7328 CNRS - Université d'Orléans, France

${ }^{11}$ Swedish Institute of Space Physics, Ångström Laboratory, Lägerhyddsvägen 1, Uppsala, Sweden.

${ }^{12} \mathrm{TU}$ - Braunschweig, Institute for Geophysics and extraterrestrial Physics, Mendelssohnstr. 3, D-38106 Braunschweig, Germany.

${ }^{13}$ Wigner Research Centre for Physics, 1121 Konkoly Thege street 29-33, Budapest, Hungary.

${ }^{14}$ Space Research Institute, Austrian Academy of Sciences, Schmiedlstraße 6, 8042 Graz, Austria

${ }^{15}$ Rosetta Science Ground Segment, SRE-OOR, Office A006, European Space Astronomy Centre, P.O. Box 78, 28691 Villanueva de la Cañada, Madrid, Spain.

${ }^{16}$ Physikalisches Institut, University of Bern, Sidlerstr. 5, CH-3012 Bern, Switzerland.

*Correspondence to: hans.nilsson@irf.se

Abstract: The Rosetta mission has been designed to rendezvous with and escort comet 67P/Churyumov-Gerasimenko from a heliocentric distance of $>3.6 \mathrm{AU}$, when the comet still has a low activity level, until perihelion passage at $1.25 \mathrm{AU}$ where the comet reaches the maximum 
of its activity. Initially, the solar wind permeates the thin comet atmosphere that has just begun to form from sublimation of the surface material. Eventually the size and plasma pressure of the ionized atmosphere leads to the formation of plasma boundaries: a magnetosphere is born. Using the Rosetta Plasma Consortium Ion Composition Analyzer, we study the gradual evolution from the first detectable traces of water ions to the stage where the comet atmosphere starts to repel the solar wind at a distance from the sun of about 3.3 AU. We have observed the spatial structure of this early interaction between the comet atmosphere and the solar wind. The nearcomet water ion population is composed of accelerated ions of energy up to $800 \mathrm{eV}$, produced upstream of the observation point, and lower energy locally produced ions. We provide quantitative estimates of fluxes of different ion species and energetic neutral atoms in the nearcomet environment.

One Sentence Summary: Characterization of the energetic ion environment near a low activity comet nucleus resulting from solar wind - comet atmosphere interaction

\section{Main Text:}

An active comet emits gas and dust that expand into the surrounding space and form the comet atmosphere or coma. Its neutral component, mainly composed of $\mathrm{H}_{2} \mathrm{O}, \mathrm{CO}$ and $\mathrm{CO}_{2}$, is thought to result from the sublimation of surface ice or icy grains when the comet draws closer to the Sun (1). Interplanetary space is permeated by the solar wind: a stream of charged particles (plasma) emitted from the sun. The electromagnetic forces of the solar wind act on the charged particles in the environment of the comet. Charged particles are created as the atmosphere is ionized by extreme ultraviolet light as well as through collisions between the solar wind and the comet atmosphere. The partially ionized and collisional atmosphere of a well-developed comet is electrically conductive. The conductive part of the atmosphere acts as an obstacle to the solar wind. When the solar wind meets this obstacle, plasma boundaries such as a bow shock and an ionopause (contact surface) form, separating the solar wind domain from the atmospheric plasma (2-4). This resembles the interaction between the solar wind and the unmagnetized planets Mars and Venus. The sun distance where the comet-solar wind interaction changes to this more "active" stage depends on the comet. For comet 67P/Churyumov-Gerasimenko at the time of the Rosetta rendezvous the nucleus outgassing rate is low (of the order of $10^{26}$ molecules $/ \mathrm{s}$ ). The resulting comet atmosphere is thin enough that the ion - neutral collision frequency is negligible. The solar wind should be largely unaffected by the comet atmosphere, and the plasma boundaries will not yet have formed $(5,6)$.

Due to weak gravity, the neutral atmosphere of a comet extends well into the solar wind, beyond the plasma boundaries once formed. If a constituent of an atmosphere is ionized within the solar wind domain, the newborn ion is accelerated by the solar wind electric field: the ion is "picked up" by the solar wind. Newly picked-up ions may form ring distributions in velocity space, if the source region is large compared to the ion gyro radius. This is not the situation we expect at a comet with a low outgassing rate (6). The accelerated ion gains energy provided by the solar wind. If the energy taken from the solar wind becomes significant, the solar wind is slowed down noticeably and deflected in a process known as mass loading (7). Mass loading is believed to be important for planets around young stars, where the solar extreme ultraviolet radiation heating the upper part of an atmosphere is generally more intense than for an older star such as the sun (8), causing a higher temperature and an extended scale height of the upper atmosphere. More of the upper atmosphere may thus reach into the solar wind domain. Another possible situation is that the dynamic pressure of a stellar wind is significantly higher than the ionospheric plasma 
pressure of a planet (9), leading to a situation where the solar wind magnetic field permeates the entire atmosphere of the planet. A weak comet represents an example of the situation when the local plasma pressure cannot balance the solar wind dynamic pressure. The solar wind itself, as well as ion and neutral fluxes resulting from the interaction of the atmosphere and solar wind, may affect atmospheric and surface chemistry at a comet or a planet with a very thin atmosphere.

The Rosetta mission reached comet 67P/Churyumov-Gerasimenko at a heliocentric distance of about 3.6 AU when the comet was still in a low activity state. For all previous in-situ spacecraft comet encounters, the targeted comet had high activity, with an enormous atmosphere extending millions of $\mathrm{km}$ into space, and well-formed plasma boundaries with a bow shock and ionopause. On 13 March 1986 the Giotto spacecraft flew by comet Halley at a sun distance of $0.89 \mathrm{AU}$. The relative velocity was a few tens of $\mathrm{km} / \mathrm{s}$, with a closest approach distance of about $600 \mathrm{~km}$. The outgassing rate of Halley at that time was estimated to be of the order of $10^{30}$ molecules/s. The bow shock was observed at $\sim 1$ million $\mathrm{km}$ from the nucleus, and the ionopause at $\sim 5000 \mathrm{~km}(10,11)$. Rosetta thus enters a wholly different domain, approaching a low activity comet as close as $10 \mathrm{~km}$ from the nucleus and remaining in its close vicinity at a walking pace. We study this environment using the Ion Composition Analyzer, part of the Rosetta Plasma Consortium (RPC-ICA) (12,13). RPC-ICA can distinguish between different mass groups of ions with a mass per charge ratio of about $1,2,4,8,16$ and $32 \mathrm{amu} / \mathrm{e}$ and covers an energy range per charge of $10 \mathrm{eV} / \mathrm{e}$ to $40 \mathrm{keV} / \mathrm{e}$ in $12 \mathrm{~s}$. The basic field-of-view is near $2.8 \pi \mathrm{sr}$, with an electrostatic entrance elevation sweep providing $\mathrm{a} \pm 45^{\circ}$ view away from a central $360^{\circ}$ viewing plane divided into 16 sectors of $22.5^{\circ}$. Due to spacecraft blocking, the actual field-of-view is closer to $2 \pi \mathrm{sr}$ or half the sky. A complete angular scan is performed in 16 steps taking $192 \mathrm{~s}$ to complete. Due to instrument limitations the elevation field of view is restricted to only small elevation angles in the lowest energy bins. Water ions are expected to be one of the most common ions in the near vicinity of the comet $[1-5,14]$. RPC-ICA cannot distinguish water ions from $\mathrm{O}^{+}$or $\mathrm{H}_{3} \mathrm{O}^{+}$ions, but for convenience we will call ions in the mass group in the vicinity of mass $16 \mathrm{amu} / \mathrm{e}$ "water ions" throughout this paper (the mass of water molecules is $18 \mathrm{amu}$ ). Similarly, for convenience we will express energy as $\mathrm{eV}$, which is really eV/e, energy per charge.

The first detectable traces of water ions were observed on 7 August 2014, at a distance of 100 $\mathrm{km}$ from the nucleus of comet 67P/Churyumov-Gerasimenko (Fig. 1A). The sun-comet distance was 3.6 AU. The cyclical nature of the data is due to the entrance angle sweep. The solar wind ions are only seen when the instrument probes the solar wind direction. Inspection of the full data set reveals that the ions at $\sim 650 \mathrm{eV}$ are protons $\left(\mathrm{H}^{+}\right)$, those at $\sim 1300 \mathrm{eV}$ are alpha particles $\left(\mathrm{He}^{2+}\right)$ and the ions in the lower energy bins are water ions. The water ions are coming from a direction $90^{\circ}$ from that of the solar wind direction. This is consistent with the ions moving in the direction of the solar wind electric field. The water ions and the solar wind are for this event seen for the same elevation angle (same time in Fig. 1A). The lowest energy ions can only be seen for small elevation angles due to instrument limitations. The sun is always seen at low elevation angles due to constraints on spacecraft orientation imposed by the solar panels. The water ion flux for this first event was up to $3 \times 10^{8} \mathrm{~m}^{-2} \mathrm{~s}^{-1}$. With a drift velocity in the range 10 to $20 \mathrm{~km} / \mathrm{s}$ (the latter corresponding to a drift energy of about $40 \mathrm{eV}$ ), the water ion density is $\sim 10^{4}-10^{5} \mathrm{~m}^{-}$ 3. The data from 7 August are typical for the early observations, with an undisturbed solar wind. The weak water ion flux of a few $10^{8}$ ions $\mathrm{m}^{-2} \mathrm{~s}^{-1}$ does not yet influence the solar wind. 
By 11 August 2014, the water ions are accelerated to nearly $100 \mathrm{eV}$ (Fig. 1B). The fact that no or few ions are seen at the lower energies indicates that there is some distance between the spacecraft and the strongest water ion source. The energy of the ion directly corresponds to the travel distance along the solar wind electric field, which is expected to be of the order of $1 \mathrm{~V} / \mathrm{km}$ or less. The ions thus appear to have traveled several tens of $\mathrm{km}$ from a main source to the observation points.

We observed enhanced activity on 21 September 2014 (Fig. 2A). The distance to the comet center was just below $28 \mathrm{~km}$ and Rosetta was over the northern hemisphere of the comet. The sun distance was 3.3 AU. The repeating pattern of ions in the 700 to $800 \mathrm{eV}$ range is solar wind $\mathrm{H}^{+}$, at $1400-1600 \mathrm{eV}$ it is $\mathrm{He}^{2+}$, while at $3000 \mathrm{eV}$ a weak signal from $\mathrm{He}^{+}$can occasionally be seen. On this day, RPC-ICA detected solar wind fluxes about an order of magnitude higher than average, up to $10^{12} \mathrm{~m}^{-2} \mathrm{~s}^{-1}$ for $\mathrm{H}^{+}$and $10^{11} \mathrm{~m}^{-2} \mathrm{~s}^{-1}$ for $\mathrm{He}^{2+}$.

At around 18 UT we see a significant flux of water ions at the lowest energy we can measure. The observed water ion flux is $10^{10} \mathrm{~m}^{-2} \mathrm{~s}^{-1}$. The ions are accelerated into the instrument by a negative spacecraft potential, determined using the RPC-LAP instrument (15) to be -5 to $-10 \mathrm{~V}$. Fig. 3 shows the geometry of the observations and flow directions of all observed populations in the detector central plane. The solar wind is now slightly deflected due to interaction with the comet atmosphere and has a component of flow away from the comet. $\mathrm{H}^{+}$is somewhat more deflected than $\mathrm{He}^{2+}$. The coarse sector resolution makes a precise angle difficult to determine. The deflection is also seen in the higher angular resolution elevation scans: protons are typically deflected by $\pm 25^{\circ}$ in elevation angle from the sun line and $\mathrm{He}^{2+}$ by about half that angle. The elevation angle is the angle out of the plane depicted in Fig. 3.

We observe the low-energy water ions in the lowest energy bin only. An upper limit to the drift energy is thus $10 \mathrm{eV}$ or a velocity of about $10 \mathrm{~km} / \mathrm{s}$, corresponding to a water ion density of $10^{6}$ $\mathrm{m}^{-3}$. The RPC-LAP Langmuir probes (15) indicate a total plasma density of $10^{7} \mathrm{~m}^{-3}$, which is consistent with our observations if the drift velocity is $1 \mathrm{~km} / \mathrm{s}$. The neutral density determined using Rosetta Orbiter Spectrometer for Ion and Neutral Analysis Cometary Pressure Sensor (ROSINA COPS) (16) was a few times $10^{13} \mathrm{~m}^{-3}$. The ionization time for water molecules at 3.3 AU is about $10^{7} \mathrm{~s}$. Thus the observed ion density would be produced in 1 to $10 \mathrm{~s}$ over a distance of $10 \mathrm{~km}$, the order of magnitude of the distances involved. A water ion density of $10^{7} \mathrm{~m}^{-3}$ would correspond to about the same particle density as that of the solar wind $\mathrm{H}^{+}$and the mass density would be an order of magnitude higher than that of the solar wind. The kinetic energy density of water ions is still tiny compared to that of the solar wind, with a kinetic energy ratio of $10^{-4}$ to $10^{-}$ ${ }^{3}$ for 1 and $10 \mathrm{~km} / \mathrm{s}$ water ions, respectively.

We also examined the narrower time period 20:53 to 20:59 UT on 21 September 2014 (Fig. 2B). Due to instrument angular scans we see repetitive structures of accelerated ions forming curves from the lowest energy up to that of solar wind $\mathrm{H}^{+}$at $800 \mathrm{eV}$ (the ions are seen in sector 12; see Fig. 3 for a schematic overview). The ions appear to follow an angular dispersion, with higher energy ions seen for larger elevation deflection angles (seen as a time series in the energy spectrogram). Careful analysis reveals that this is an artifact caused by an offset in our energy table. All these ions are seen just at the limit of observable angles for that energy. The ions come from a small range of angles of $20^{\circ}$ to $30^{\circ}$. The ions reach energies of $800 \mathrm{eV}$, which implies acceleration over a distance of a few $100 \mathrm{~km}$ assuming that the solar wind electric field is the underlying acceleration mechanism. The integrated flux of the accelerated cometary ions is $10^{9}$ $\mathrm{m}^{-2} \mathrm{~s}^{-1}$, an order of magnitude less than the cold water ions. 
By constructing an energy mass matrix from the time period when accelerated cometary ions were observed on 21 September 2014, we see that the different ion populations are seen where they are expected, i.e. $\mathrm{H}^{+}$at mass per charge 1 , alpha particles at $2 \mathrm{amu} / \mathrm{e}, \mathrm{He}^{+}$at $4 \mathrm{amu} / \mathrm{e}$ and the accelerated ions around $16 \mathrm{amu} / \mathrm{e}$ (Fig. 4). The highest and lowest energy bins with significant fluxes of cometary ions appear also to include ions with mass of well above $16 \mathrm{amu} / \mathrm{e}$. This indicates the presence of some heavier ions $\left(\mathrm{CO}^{+}, \mathrm{CO}_{2}^{+}\right)$for this occasion.

The observed $\mathrm{He}^{+}$are created by charge exchange between $\mathrm{He}^{2+}$ and $\mathrm{H}_{2} \mathrm{O}$ and allow us to make an estimate of the total $\mathrm{H}_{2} \mathrm{O}$ atmosphere the ions have passed through, i.e., $2.5 \times 10^{17} \mathrm{~m}^{-2}$ (17). This corresponds to an in-situ neutral $\mathrm{H}_{2} \mathrm{O}$ density of about $1 \times 10^{13} \mathrm{~m}^{-3}$, in good agreement with ROSINA COPS measurements (16). The charge exchange of $\mathrm{H}^{+}$into $\mathrm{H}$ is more important for the near-comet environment, as this constitutes a much larger flux. It has commonly been observed at other solar system objects such as Mars (18). RPC-ICA cannot measure these energetic neutral atoms, but we can infer the approximate flux of $\mathrm{H}$ by comparing with the effect of the charge exchange product that we can observe, i.e., $\mathrm{He}^{+}$. The flux ratio of $\mathrm{He}^{+}$to $\mathrm{He}^{2+}$ for 30 September 2014, the day when we observed the clearest $\mathrm{He}^{+}$signal, was $2.1 \%$ (17). Using models of charge exchange, we then estimate that at the same point in space we should have a $\mathrm{H}$ to $\mathrm{H}^{+}$ratio of $4.4 \%$. Scaling this value with a model of the comet atmosphere density, we find that the $\mathrm{H}^{+} \mathrm{H}^{+}$ ratio close to the nucleus reaches up to $33 \%$ at the terminator, i.e., a large fraction of the impinging solar wind will have charge-exchanged with water molecules close to the nucleus, leading to the formation of a relatively dense cloud of energetic neutral hydrogen atoms.

At $100 \mathrm{~km}$ from a low-activity comet nucleus, where we first detected the traces of cometary water ions, the source of the observed ions is already spatially structured. This is revealed through the absence of ion populations at the lowest energies. Thus at $100 \mathrm{~km}$ from the nucleus, the number of locally produced ions was below the measurement threshold of the instrument. This view is supported by time variations of the local neutral density as measured by the ROSINA instrument which measures significant variations related to the comet nucleus rotation period (19).

At a distance of $28 \mathrm{~km}$ from the nucleus, we observe a comet plasma of similar number density to that of the solar wind. The neutral atmosphere had a density of $10^{13} \mathrm{~m}^{-3}$, similar to the $\mathrm{F}$ region in Earth's ionosphere (20). This atmosphere is still essentially collision-less, but chargeexchange collisions are already noticeable. We are witnessing the initial stages of an ionosphere being born.

In this nascent stage, before the plasma boundaries are formed, the comet nucleus and nearcomet atmosphere are affected by a relatively direct flux of solar wind. However at this stage, the solar wind is already somewhat deflected by the comet atmosphere. The deflection is about $20^{\circ}$ from the sun-comet line for $\mathrm{H}^{+}$and about half of that for $\mathrm{He}^{2+}$. Simple calculations indicate that a large fraction of the solar wind impinging on the nucleus and lowest part of the atmosphere will undergo charge exchange: $30 \%$ for our example day of 30 September 2014. Energetic cometary ions were observed at $28 \mathrm{~km}$ from the nucleus. The continuous presence of ions throughout the 
energy interval from near 0 to $800 \mathrm{eV}$ indicates a continuous source extending over a few 100 $\mathrm{km}$. Thus similar fluxes can be expected to hit the nucleus. Based on our observations, the nucleus is consequently [?] hit by the solar wind, by charge-exchanged $\mathrm{H}$ and a flux of $10^{9} \mathrm{~m}^{-2} \mathrm{~s}$ ${ }^{1}$ of accelerated ions with a cometary origin, with an energy of up to $800 \mathrm{eV}$ per charge. The neutral hydrogen flux will not be affected by the enhanced magnetic field caused by the solar wind slowing down and by associated magnetic field line draping. The important role of a heterogeneous and dynamic comet atmosphere, the deflection of the solar wind at an early stage, and the presence of accelerated ions - these present challenges for comet-solar wind interaction models $(6,21)$. This energetic ion environment represents the first stage of the birth of the comet magnetosphere.

\section{References and Notes:}

1. Altwegg, K., H. Balsiger, and J. Geiss, Composition of the volatile material in Halley's coma from in situ measurements, Space Sci. Rev. 90, 3-18, (1999).

2. T. Mukai, W. Miyake, T. Terasawa, M. Kitayama, K. Hirao, Plasma observation by Suisei of solar-wind interaction with comet Halley, Nature 321,299-303 (1986).

3. F. M. Neubauer, Giotto magnetic field results on the boundaries of the pile-up region and the magnetic cavity, Astron. Astrophys. 187,73-79 (1987).

4. K. I. Gringauz et al., First in situ plasma and neutral gas measurements at comet Halley, Nature 321, 282-285 (1986).

5. A. J. Coates, Ionospheres and magnetospheres of comets, Adv. Space Res. 20, No. 2, 255266 (1997).

6. M. Rubin et al., Plasma environment of a weak comet - Predictions for Comet 67P/Churyumov-Gerasimenko from multifluid-MHD and Hybrid models, Icarus 242, 38-49 (2014).

7. K. Szego et al., Physics of mass loaded plasmas, Space Sci. Rev. 94, 429-671 (2000).

8. I. Ribas et al., Evolution of the solar activity over time and effects on planetary atmospheres. I. High-energy irradiances (1-1700 Å), Astrophys. J. 622, 680-694 (2005).

9. J. G. Luhmann, The solar wind interaction with unmagnetized planets: a tutorial, Geophysical Monograph, 58 401-411 (1991).

10. R. Reinhard, The Giotto Encounter with Comet Halley. Nature 321 313-318 (1986).

11. A. Johnstone et al., Ion flow at comet Halley, Nature, 321 344-347 (1986).

12. C. Carr et al., RPC: The Rosetta plasma consortium, Space Sci. Rev., 128 629-647 (2007).

13. H. Nilsson et al., RPC-ICA: The ion composition analyzer of the Rosetta plasma consortium, Space Sci. Rev., 128 671-695 (2007).

14. A. J. Coates et al. Pick up water ion groups at comet Grigg-Skjellerup, Geophys. Res. Lett., 20 483-486 (1993)

15. A. Eriksson et al., RPC-LAP: The Rosetta Langmuir probe instrument, Space Sci. Rev., 128 729-744 (2007).

16. H. Balsiger et al., Rosina - Rosetta orbiter spectrometer for ion and neutral analysis, Space Sci. Rev., 128 745-801 (2007).E. Kallio. et al., Energetic Neutral Atoms (ENA) at 
Mars: Properties of the hydrogen atoms produced upstream of the martian bow shock and implications for ENA sounding technique around non-magnetized planets, Icarus 182,(2), 448-463, doi:10.1016/j.icarus.2005.12.019, (2006).

17. Supplementary Material, Calculation of ENA fluxes

18. E. Kallio et al., Energetic Neutral Atoms (ENA) at Mars: Properties of the hydrogen atoms produced upstream of the martian bow shock and implications for ENA sounding technique around non-magnetized planets, Icarus 182(2), 448-463, doi:10.1016/j.icarus.2005.12.019, (2006).

19. M. Hässig et al. Time variability and heterogeneity in the coma of 67P/ChuryumovGerasimenko, Science this issue.

20. M. H. Rees, Physics and chemistry of the upper atmosphere (Cambridge University Press, 1989).

21. K. C. Hansen et al., The Plasma Environment of Comet 67P/Churyumov-Gerasimenko Throughout the Rosetta Main Mission, Space Science Reviews 128, p. 133-166 (2007).

22. J.B. Greenwood et al., The Contribution of Charge Exchange to Extreme Ultra-Violet and X-ray Astronomy, Phys. Scr. T 110, 358-363 (2004).

23. L. Haser, Distribution d'intensité dans la tête d'une comète. Bull. Soc. Roy. Sci. Liège 43, 740-750 (1957).

24. B.G. Lindsay et al., Charge transfer of 0.5-, 1.5-, and 5-keV protons with $\mathrm{H}_{2} \mathrm{O}$ : Absolute differential and integral cross sections, Phys. Rev. A 55, 3945-3946 (1997).

Acknowledgments: Rosetta is a European Space Agency (ESA) mission with contributions from its member states and the National Aeronautics and Space Administration (NASA). Rosetta's Philae lander is provided by a consortium led by DLR, MPS, CNES and ASI. The work on RPC-ICA was funded by the Swedish National Space Board under contracts 108/12 and 112/13. We are indebted to the whole Rosetta mission team, Science Ground Segment and Rosetta Mission Operation Control for their hard work making this mission possible. The RPC data is available through the PSA archive of ESA and the PDS archive of NASA.

Figure 1. Energy-time ion spectrograms: (A) Data obtained on 7 August 2014 showing the first detection by RPC-ICA of water ions from the comet. The color scale shows differential flux summed over all azimuthal sectors and all masses. The water ions are the signal at just above $10 \mathrm{eV}$. Solar wind proton and alpha particles are visible at higher energies $(650 \mathrm{eV}, 1300 \mathrm{eV})$. The black patches indicate times of decoder errors; (B) Energy-time spectrogram from 11 August 2014. The water ions are accelerated to nearly $100 \mathrm{eV}$. 
Figure 2 Energy-time ion spectrograms: (A) Data from 21 September 2014. The color scale is differential flux. The solar wind ions are visible at the $700-800 \mathrm{eV}\left(\mathrm{H}^{+}\right)$and $1400-1600 \mathrm{eV}$ $\left(\mathrm{He}^{2+}\right)$. At even higher energies $(3000 \mathrm{eV})$ a weak signal from $\mathrm{He}^{+}$is sometimes seen. In the lowest energy bin we see water ions; (B) Details of the time period 20:53 to 20:59 on 21 September. The solar wind components are clearly seen at different energies with a faint $\mathrm{He}^{+}$ signal at higher energy. The ions seen in the energy range 100-1000 eV, forming curves, are comet origin water ions with an elevation angle of $20^{\circ}$ to $30^{\circ}$.

Figure 3. The geometry of the RPC-ICA observations made on 21 September 2014. The cold ions were mainly observed between 17 and 19 UT. The accelerated ions were observed between 21:00 and 21:30 UT. Sector numbers identify the field-of-view of the corresponding instrument sector. Comet shape model credit: ESA/Rosetta/NAVCAM.

Figure 4. Energy-mass matrix from 21 September 2014. . The observed counts are integrated over a time period including the data shown in Fig 2(B). The white lines show the position of the peak of the signal for ions with mass $1 \mathrm{amu} / \mathrm{e}$ (dotted), $2 \mathrm{amu} / \mathrm{e}$ (dashed), $4 \mathrm{amu} / \mathrm{e}$ (solid) and 16 amu/e (dash-dotted), respectively. $\mathrm{H}^{+}, \mathrm{He}^{2+}$ and $\mathrm{He}^{+}$are seen at about 750,1500 and $3000 \mathrm{eV}$. Cometary ions are seen at $10 \mathrm{eV}$. Grey lines show extrapolation beyond $300 \mathrm{eV}$, the lowest energy available from laboratory calibration. The line at mass about $16 \mathrm{amu} / \mathrm{e}$ has been determined through in-flight calibration. The signal at $16 \mathrm{amu} / \mathrm{e}$ has an asymmetric shape with the peak to the right and a rather sharp cut-off towards higher mass channels. This is observed both in the laboratory and in flight.

\section{Supplementary Materials:}

\section{Instrument Operations}

\section{Basic Theory}

Field of View of the Instrument

\section{Calculation of ENA fluxes}

Figures S1

Table S1

References (22-24) 